\title{
Curative resection for leiomyosarcoma of the descending mesocolon with metachronous liver metastasis: A case report and literature review
}

\author{
TAKAHARU KATO ${ }^{1,2}$, HIROSHI NODA ${ }^{1}$, IKU ABE $^{1}$, SERGIO ALONSO $^{2}$, \\ NOBU YOKOYAMA $^{1}$ and TOSHIKI RIKIYAMA ${ }^{1}$ \\ ${ }^{1}$ Department of Surgery, Saitama Medical Center, Jichi Medical University, Saitama, Saitama 330-8503, Japan; \\ ${ }^{2}$ Institute of Predictive and Personalized Medicine of Cancer (IMPPC), Health Sciences Research Institute \\ Germans Trias i Pujol (IGTP), Campus Can Ruti, 08916 Badalona, Barcelona, Spain
}

Received December 17, 2015; Accepted April 7, 2016

DOI: $10.3892 / \mathrm{mco} .2016 .874$

\begin{abstract}
Mesenteric leiomyosarcoma is a rare disease with poor prognosis. Previously, mesenteric leiomyosarcoma was not differentiated from gastrointestinal stromal tumor (GIST), which is the most common mesenchymal tumor of the gastrointestinal tract, and several cases of GIST may have been misclassified as mesenteric leiomyosarcoma. Thus, the actual clinicopathological characteristics of mesenteric leiomyosarcomas remain unclear. We herein describe a case of leiomyosarcoma arising in the descending mesocolon in a patient who developed metachronous liver metastasis. A 76-year-old woman reported a mass in her left upper abdomen. Computed tomography imaging revealed a low-density tumor adjacent to the descending colon. The patient underwent surgery and the tumor was resected along with part of the descending colon. Immunohistochemical differential diagnosis revealed that the tumor was positive for smooth muscle actin and desmin, and negative for CD117 (c-KIT) and S-100, which are characteristic of gastrointestinal leiomyosarcoma. A single liver metastasis developed 24 months after the operation. The patient underwent curative resection of the metastatic lesion. Sixteen months following surgery for the liver metastasis and 40 months after the initial removal of the primary lesion, the patient remains disease-free. The prognosis of leiomyosarcoma remains poor and standardized chemotherapy for this rare disease has not yet been established. Early diagnosis and
\end{abstract}

Correspondence to: Dr Takaharu Kato, Department of Surgery, Saitama Medical Center, Jichi Medical University, 1-847 Amanuma-cho, Omiya-ku, Saitama, Saitama 330-8503, Japan E-mail: tkato@jichi.ac.jp

Abbreviations: GIST, gastrointestinal stromal tumor; IHC, immunohistochemical; CT, computed tomography; FDGPET, fluorodeoxyglucose positron emission tomography; MRI, magnetic resonance imaging

Key words: mesocolon, leiomyosarcoma, liver metastasis, surgical resection, immunohistochemical analysis surgical removal of the tumor is the only potentially curative option for liver metastasis of mesenteric leiomyosarcoma.

\section{Introduction}

Mesenteric leiomyosarcoma is a rare disease with poor prognosis. Since 1998, it is acknowledged that the proper diagnosis of leiomyosarcoma requires immunohistochemical (IHC) analysis to differentiate it from gastrointestinal stromal tumor (GIST) (1), which is the most common mesenchymal tumor of the gastrointestinal tract. Several GISTs diagnosed before 1998 were misclassified as leiomyosarcomas; therefore, the frequency of leiomyosarcoma may have been previously overestimated. The clinical and pathological characteristics of mesenteric leiomyosarcoma have not been clearly determined. In this report, we present the case of a patient who underwent curative surgery for leiomyosarcoma arising in the descending mesocolon and for a metachronous liver metastasis, together with the clinicopathological findings and a literature review.

\section{Case report}

A 76-year-old woman noticed a mass in her left upper abdomen and consulted her local clinic, where a $12-\mathrm{cm}$ abdominal tumor was detected by ultrasonographic evaluation. The patient was referred to the Saitama Medical Center, Jichi Medical University (Saitama, Japan) for further investigation and treatment. The patient's past medical history included hepatitis $\mathrm{C}$ infection, an ovarian cyst and appendicitis. The cyst and the appendix were surgically resected at the age of 4 and 34 years, respectively. The patient had a family history of cancer, as her mother had developed colorectal cancer. Physical examination revealed a palpable mass, sized $\sim 5 \mathrm{~cm}$, in the left upper abdomen, which was elastic hard and movable. No lymph nodes were palpable. The patient did not suffer from nausea. The tumor markers were marginally elevated (carcinoembryonic antigen, $5.6 \mathrm{ng} / \mathrm{ml}$ and carbohydrate antigen 19-9, $22.2 \mathrm{U} / \mathrm{ml}$ ). Interleukin-2 receptor level was within the normal range. Abdominal computed tomography $(\mathrm{CT})$ revealed a $13-\mathrm{cm}$ tumor, heterogeneously 


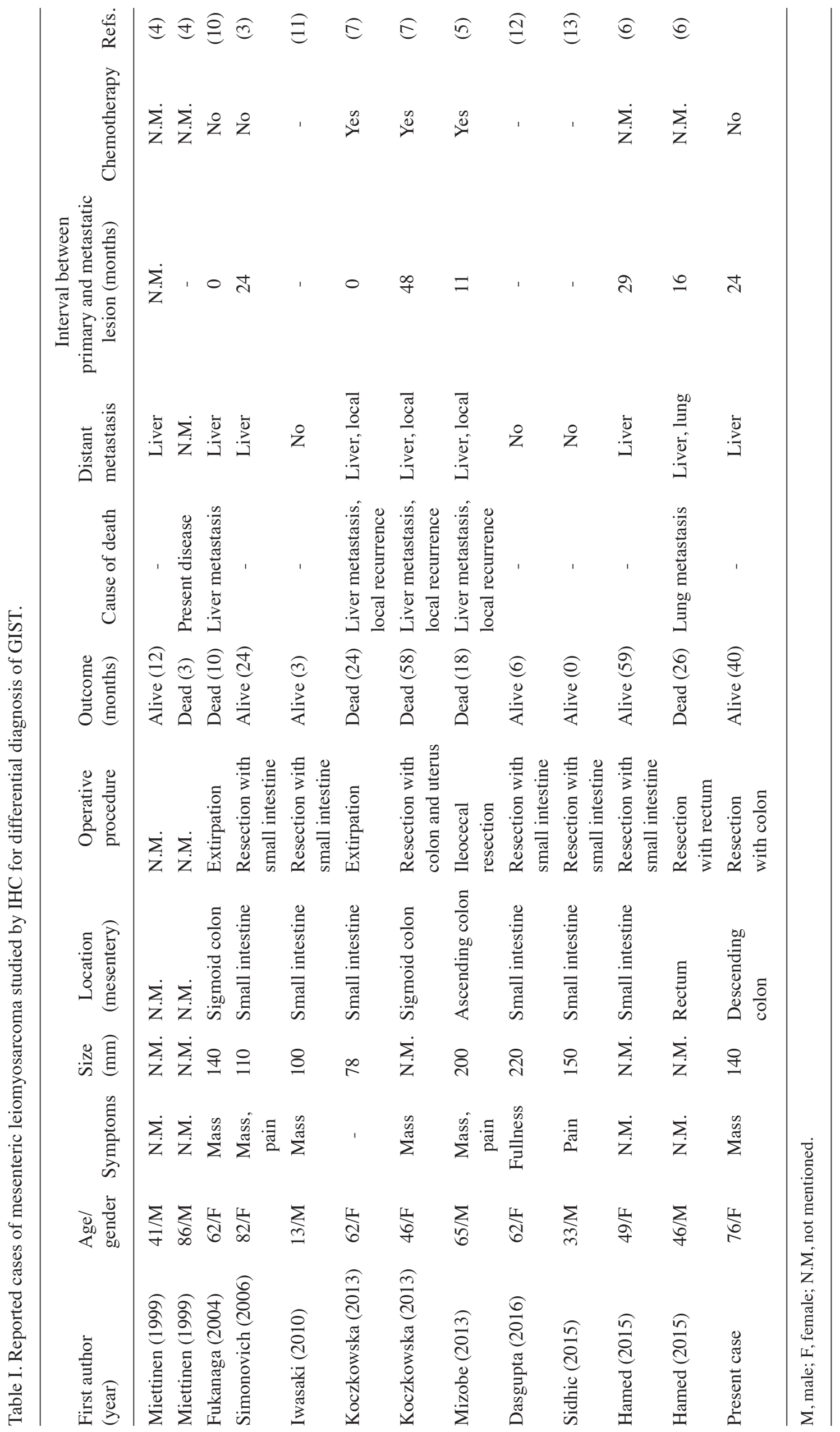




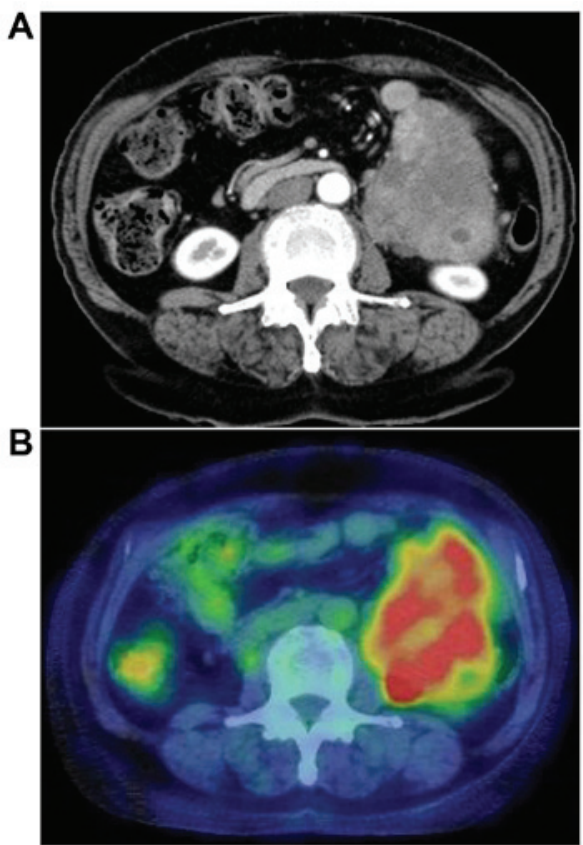

Figure 1. (A) Abdominal computed tomography (CT) revealed an irregular mass enhanced heterogeneously in the left lower abdomen, sized 9x7 cm (B) A fluorodeoxyglucose (FDG) positron emission tomography/CT fusion image revealed a tumor in the left lower abdomen with significantly increased FDG uptake (maximum standardized uptake value $=5.6$ ).

enhanced, located in the abdominal cavity between the tail of the pancreas and the anterior surface of the left kidney. The margin with the surrounding organs was clear, except for the small intestine. There was no evidence of ascites, liver metastasis, or enlarged lymph nodes (Fig. 1A). The tumor was fluorodeoxyglucose (FDG)-avid on FDG-positron emission tomography (PET)/CT (Fig. 1B). Colonoscopy revealed no evidence of a tumor in the descending colon. The patient was diagnosed with a tumor arising in the retroperitoneal or descending mesocolon and underwent surgery in June, 2012. Intraoperatively, the tumor was located in the descending mesocolon, it was movable and easily excised from the left psoas muscle and urinary duct. However, the tumor invaded the mesenteric vein; therefore, en bloc removal of the descending colon was performed. Macroscopically, the size of the tumor was $14 \times 9 \times 9 \mathrm{~cm}$, it weighed $500 \mathrm{~g}$, its surface was smooth and elastic hard, and the cut surface was solid and white (Fig. 2A). The histological findings included high cellularity, spindle-shaped cells with blunted hyperchromatic nuclei, exhibiting a trabecular proliferation pattern that indicated differentiation towards smooth muscle (Fig. 2B). IHC analysis revealed that the tumor was positive for smooth muscle actin and desmin (Fig. 2C and D) and negative for CD117 (c-KIT) and S-100. From these findings, the final diagnosis was confirmed as leiomyosarcoma of the descending mesocolon. The patient's postoperative course was uncomplicated and she was discharged from the hospital 11 days after surgery. She was followed up every 6 months by blood tests and imaging studies [CT, magnetic resonance imaging (MRI) and ultrasonography] and did not receive any adjuvant chemotherapy. A tumor at segment VI in the right lobe of the liver was detected 24 months after the surgery.
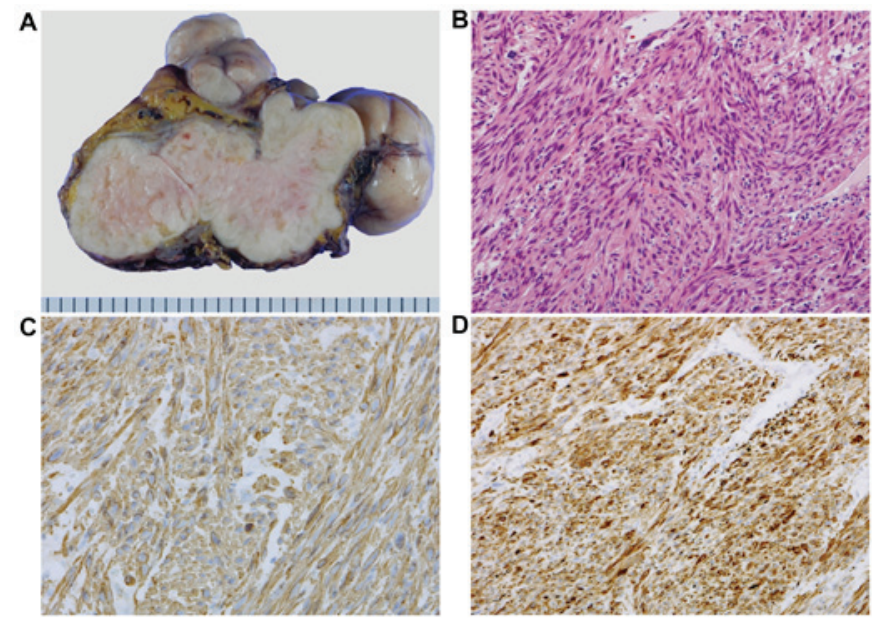

Figure 2. (A) The resected tumor was solid and white-yellowish, sized 140x90x90 mm, containing several lobules. The cut surface was white and elastic hard. (B) The histological examination of the resected specimen revealed atypical spindle cell proliferation, indicating differentiation towards smooth muscle (hematoxylin-eosin staining; magnification, $\mathrm{x} 200$ ). On immunohistochemistry, the tumor cells stained positive for (C) $\alpha$ smooth muscle actin and (D) desmin (magnification, x200), but negative for CD117 (c-KIT) and S-100 (not shown).
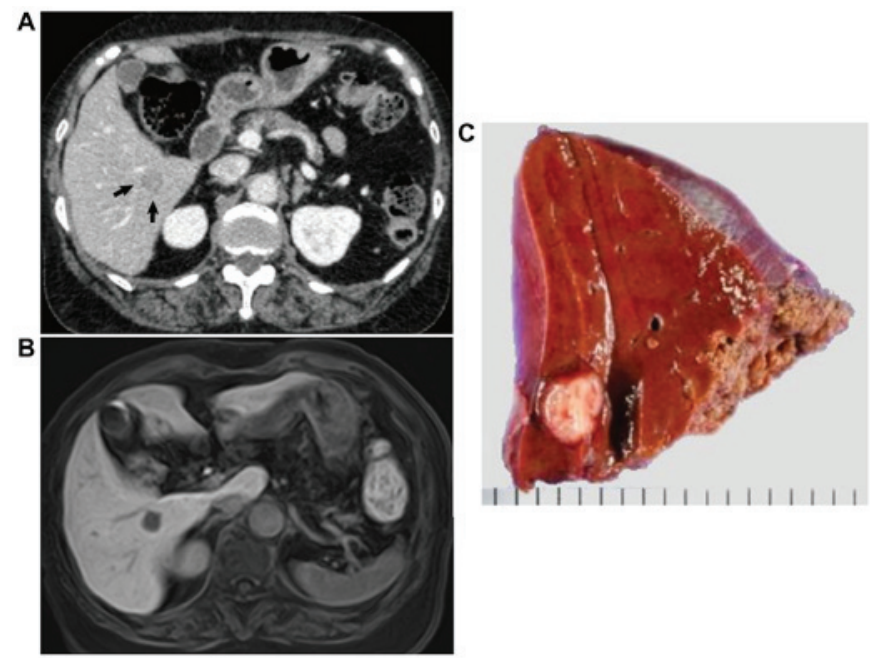

Figure 3. (A) Abdominal computed tomography revealed a 15-mm tumor at segment VI in the liver (arrows). (B) The tumor was detected as a hypointense lesion on gadolinium ethoxybenzyl diethylenetriamine pentaacetic acid-enhanced magnetic resonance imaging with a T1-weighted image of the hepatobiliary phase at segment VI of the liver. (C) The resected specimen from the liver exhibited a white nodular tumor, sized 10x10 mm.

The tumor size was $\sim 15 \mathrm{~mm}$ and marginally enhanced on CT (Fig. 3A). The tumor was also detected as a hypointense lesion on gadolinium ethoxybenzyl diethylenetriamine pentaacetic acid-enhanced MRI with a T1-weighted image of the hepatobiliary phase (Fig. 3B), but not detected on FDGPET/CT. The patient underwent partial liver resection to remove the tumor. The tumor was measured to be $12 \times 10 \mathrm{~mm}$ (Fig. 3C) and was diagnosed as a metastatic lesion from the leiomyosarcoma of the descending mesocolon by pathological examination. At the most recent follow-up, 16 months after the surgery for liver metastasis and 40 months after the primary surgery, the patient remains disease-free. 


\section{Discussion}

We herein report a case who underwent surgery for primary leiomyosarcoma of the descending mesocolon and a single metachronous liver metastasis. Mesenteric leiomyosarcoma is a rare malignancy. The first mesenteric leiomyosarcoma case in the literature was reported by Derechin et al (1) in 1956. Proper diagnosis of leiomyosarcoma requires IHC to differentiate this type of lesion from GIST, the most common mesenchymal tumor of the gastrointestinal tract. However, older reports on mesenteric smooth muscle tumors cannot be included in the current classification, since, at the time, the studies did not differentiate between leiomyosarcoma and GIST. Thus, several GISTs may have been misclassified as leiomyosarcomas; therefore, the frequency of leiomyosarcoma may have been previously overestimated. We searched the PubMed database using the key words 'leiomyosarcoma AND (mesentery OR mesenteric)', excluding articles that were published prior to 1998, which was the year the GIST concept was introduced (2). A total of 163 articles were initially identified and we eliminated reports that did not apply IHC for the differential diagnosis of GIST or reports that were not written in English. Including our case, only 13 patients have been reported to be diagnosed with mesenteric leiomyosarcoma (Table I). The median age of the patients was 62 years (range, 13-82 years) and they included 6 men and 7 women. The most common site developing leiomyosarcoma was the mesentery of the small intestine ( 6 cases) followed by the mesocolon ( 4 cases) and mesorectum ( 1 case). The remaining 2 cases were described as just mesentery, with no more specific location information. Of the 12 cases, 11 had follow-up information, with a median of 18 months (range, 3-59 months). Surgical resection with a wide margin of normal tissue is the most effective treatment strategy for mesenteric leiomyosarcoma (3). All 13 cases received surgery for the primary lesion; 6 patients succumbed to the disease during the follow-up period (3-58 months). No patient was reported to survive for $>59$ months. Of the patients who succumbed to the disease, 1 died with the originally diagnosed leiomyosarcoma (no information on surgical intervention was reported); 4 had developed liver metastasis, with $(n=3)$ or without $(n=1)$ local recurrence after surgical resection of the primary lesion; and 1 patient succumbed to lung metastasis after removal of the primary lesion (Table I). Liver is the most common organ in which metastatic lesions developed among these cases. Of note, all the patients who were followed up after surgery for $>6$ months developed liver metastasis synchronously $(\mathrm{n}=2)$ or metachronously $(\mathrm{n}=6)$. One case reported by Miettinen et al (4) developed a liver metastasis at an undetermined time after the operation and during the12-month follow-up. The median interval for developing liver metastasis after primary surgery was 20 months (range, $0-48$ months). The majority of these liver metastases were multiple, with 1 case developing metastases both in the liver and lung. Only 2 patients, including the one reported in this study, developed single liver metastasis (5). Three cases, including the patient reported in our study, underwent surgery for liver metastasis (6), and 3 cases received chemotherapy for the metastatic lesions $(5,7)$. There is no mention of surgical intervention or chemotherapy treatment for the secondary liver lesions in the remaining 3 cases. Some reports demonstrated that doxorubicin-containing chemotherapy improved the outcome of patients with metastatic leiomyosarcoma $(8,9)$. Although these reports did not include patients with mesenteric leiomyosarcoma, due to its rarity, doxorubicin-containing chemotherapy may also be effective in reducing the incidence of metastasis or recurrence of mesenteric leiomyosarcoma. However, a standardized chemotherapy regimen for metastasis or recurrence of this type of tumor is not yet established. In our case, 40 months after the initial removal of the primary lesion, the patient remains disease-free. The favorable postoperative course over an extended period of time was achieved by curative resection of both the primary tumor and the metachronous liver metastasis, without adjuvant chemotherapy. Albeit the current literature is limited due to the low incidence of mesenteric leiomyosarcomas, the data reveal a high incidence of liver metastasis in these patients. We recommend periodic monitoring using imaging techniques following surgical resection, as it would be highly beneficial in the clinical management of mesenteric leiomyosarcoma patients, facilitating early detection and curative resection of metachronous liver metastases. Further studies are required to elucidate the clinical and pathological characteristics of mesenteric leiomyosarcoma patients.

\section{References}

1. Derechin W and Wolfe S: Leiomyosarcoma of the mesentery. Can Med Assoc J 75: 1028-1029, 1956.

2. Hirota S, Isozaki K, Moriyama Y, Hashimoto K, Nishida T, Ishiguro S, Kawano K, Hanada M, Kurata A, Takeda M, et al: Gain-of-function mutations of c-kit in human gastrointestinal stromal tumors. Science 279: 577-580, 1998.

3. Simonovich CJ, Hardman JM, Navin JJ, Jacobs J and Fergusson N: An unusual abdominal tumor - leiomyosarcoma of the mesentery: A case report. Hawaii Med J 65: 18-20, 2006.

4. Miettinen M, Monihan JM, Sarlomo-Rikala M, Kovatich AJ, Carr NJ, Emory TS and Sobin LH: Gastrointestinal stromal tumors/smooth muscle tumors (GISTs) primary in the omentum and mesentery: clinicopathologic and immunohistochemical study of 26 cases. Am J Surg Pathol 23: 1109-1118, 1999

5. Mizobe T, Akagi Y, Ishikawa H, Shiratsuchi I, Oka Y, Kinugasa T, Ohshima K, Setojima K and Shirouzu K: Gemcitabine with paclitaxel therapy against mesocolic leiomyosarcoma: A case report. Anticancer Res 33: 2929-2933, 2013.

6. Hamed MO, Roberts KJ, Merchant W and Lodge JP: Contemporary management and classification of hepatic leiomyosarcoma. HPB (Oxford) 17: 362-367, 2015.

7. Koczkowska M, Lipska BS, Grzeszewska J, Limon J, Biernat W and Jassem J: Primary leiomyosarcoma of the mesentery in two sisters: Clinical and molecular characteristics. Pol J Pathol 64: 59-63, 2013.

8. Maki RG, Wathen JK, Patel SR, Priebat DA, Okuno SH, Samuels B, Fanucchi M, Harmon DC, Schuetze SM, Reinke D, et al: Randomized phase II study of gemcitabine and docetaxel compared with gemcitabine alone in patients with metastatic soft tissue sarcomas: Results of sarcoma alliance for research through collaboration study 002 (corrected). J Clin Oncol 25: 2755-2763, 2007.

9. Penel N, Italiano A, Isambert N, Bompas E, Bousquet G and Duffaud F; French Sarcoma Group (Groupe Sarcome Français/Groupe d'Etude des Tumeurs Osseuses): Factors affecting the outcome of patients with metastatic leiomyosarcoma treated with doxorubicin-containing chemotherapy. Ann Oncol 21: 1361-1365, 2010.

10. Fukunaga M: Neuron-specific enolase-producing leiomyosarcoma of the mesentery. APMIS 112: 105-108, 2004.

11. Iwasaki M, Kitaguchi K and Kobayashi H: Mesenteric leiomyosarcoma in a 13-year-old boy. J Pediatr Surg 45: 1893-1895, 2010.

12. Dasgupta S, Chakrabarti S, Ghosh S and Das S: Ileal Mesenteric Leiomyosarcoma-Report of a Rare Neoplasm. J Gastrointest Cancer 47: 114-117, 2016.

13. Sidhic AK, Ranjith M, Ali KP and Tej PR: Leiomyosarcoma of the mesentry, a rare mesentric tumour. Int J Surg Case Rep 7C: 58-60, 2015. 